Check for updates

Cite this: J. Mater. Chem. A, 2017, 5 , 15983

DOI: $10.1039 / \mathrm{c} 7 \operatorname{ta} 90141 \mathrm{k}$

www.rsc.org/MaterialsA

\title{
Correction: Roadmap and roadblocks for the band gap tunability of metal halide perovskites
}

\author{
E. L. Unger, ${ }^{\star a b}$ L. Kegelmann, ${ }^{c}$ K. Suchan, ${ }^{a}$ D. Sörell, ${ }^{a}$ L. Korte ${ }^{c}$ and S. Albrecht ${ }^{d}$
}

Correction for 'Roadmap and roadblocks for the band gap tunability of metal halide perovskites' by E. L. Unger et al., J. Mater. Chem. A, 2017, 5, 11401-11409.

The authors regret that for the data set of $\mathrm{Cs}_{y}\left(\mathrm{MA}_{0.17} \mathrm{FA}_{0.83}\right) \mathrm{Pb}\left(\mathrm{Br}_{0.17} \mathrm{I}_{0.83}\right)_{3}$, erroneous values for the lattice constants $a_{0}$ were used, causing the data points to deviate from the expected trend in the initial plot of absorption onset $v s$. pseudo-cubic lattice constant $a_{0}$. The mistake was noticed when reviewing this data set. The data points indeed fall exactly on the expected trend line and hence present a confirmation rather than deviation from the rule.

This means that the previous statement regarding this data set: "Some of the compounds of the triple-cation $\left(\mathrm{MA}_{y} \mathrm{FA}_{1-y}\right)_{1-z} \mathrm{Cs}_{z}$ $\mathrm{PbI}_{3}$ alloys (Fig. 2, brown/white square) introduced by Saliba et al. (ref. 3 in the original article) exhibit a similar offset compared to the $\operatorname{CsPb}\left(\mathrm{Br}_{x} \mathrm{I}_{1-x}\right)_{3}$ series suggesting that Cs inclusion on the A-site of the lattice affects the band gap in a comparable manner" was a misinterpretation.

The authors would hereby like to make the following statement:

"The triple-cation alloys $\left(\mathrm{MA}_{y} \mathrm{FA}_{1-y}\right)_{1-z} \mathrm{Cs}_{z} \mathrm{PbI}_{3}$ (Fig. 2, brown/white squares) introduced by Saliba et al. (ref. 3 in the original article) exhibit a similar trend as the data sets of hybrid perovskite materials, suggesting that Cs inclusion on the A-site of the lattice affects the band gap in a comparable manner."

The authors would like to replace Fig. 2 with the corrected version, shown below.

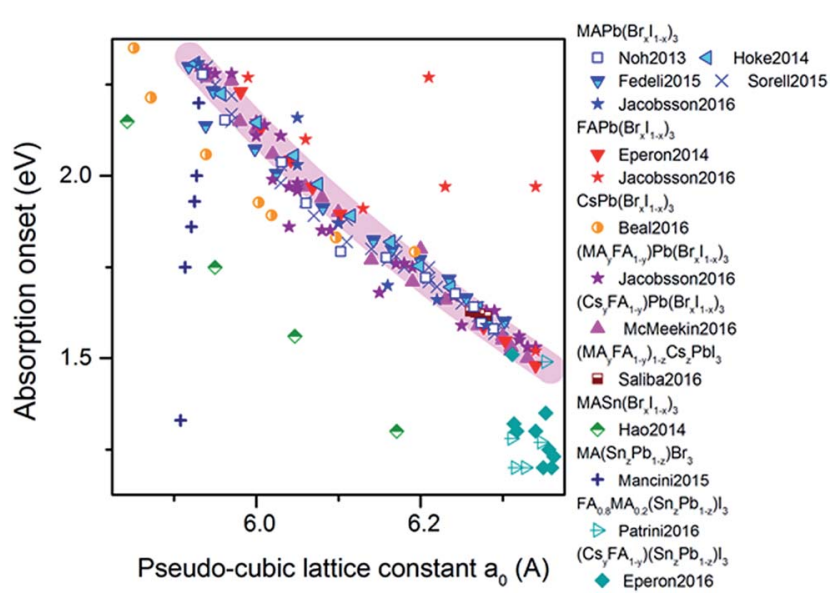

Fig. 2 The dependence of the band gap (absorption onset) on the pseudo-cubic lattice parameter $a_{0}$ for several experimental datasets. Data shown here were derived from the experimental data from ref. 3, 9-13, 17, 18, 26, 35, 45, and 46 in the original article (for detailed copyright statements please refer to the Copyright statements section in the original article).

The Royal Society of Chemistry apologises for these errors and any consequent inconvenience to authors and readers.

${ }^{a}$ Chemical Physics and NanoLund, Lund University, Box 124, SE-22100, Lund, Sweden. E-mail: eva.unger@chemphys.lu.se

${ }^{b}$ Helmholtz-Zentrum Berlin für Materialien und Energie GmbH, Young Investigator Group Hybrid Materials Formation and Scaling, Kekuléstraße 5, 12489 Berlin, Germany. E-mail: eva.unger@helmholtz-berlin.de

'Helmholtz-Zentrum Berlin für Materialien und Energie GmbH, Institut für Silizium-Photovoltaik, Kekuléstraße 5, 12489 Berlin, Germany

${ }^{d}$ Helmholtz-Zentrum Berlin für Materialien und Energie GmbH, Young Investigator Group Perovskite Tandem Solar Cells, Kekuléstraße 5, 12489 Berlin, Germany 